Cuadernos de Filología Clásica. Estudios Latinos

ISSN: 1131-9062

http://dx.doi.org/10.5209/CFCL.60934

\title{
Las Sententiae de Publilio Siro seleccionadas por Erasmo y su influencia en los florilegios de G. Maior y A. Rodrigues de Évora ${ }^{1}$
}

\author{
Francisco Bravo de Laguna Romero ${ }^{2}$; Gregorio Rodríguez Herrera ${ }^{3}$
}

Recibido: 6 de marzo de 2017 / Aceptado: 12 de enero de 2018

Resumen. La edición de Erasmo de las Sententiae de Publilio Siro (1514) marca un antes y un después en la fortuna literaria del mimo romano y su obra. Este trabajo estudia la vinculación de las sentencias de Publilio Siro, seleccionadas por Erasmo, con las Sententiae ueterum poetarum (1534) de G. Maior y las Sententiae et Exempla ex probatissimis scriptoribus collecta (1557) de A. Rodrigues de Évora. A partir del análisis de estas obras ofrecemos una clasificación temática de la selección erasmiana y demostramos el grado de seguimiento de la edición erasmiana por parte de los compiladores.

Palabras Clave: Publilio Siro; sententiae; florilegios; Erasmo.

\section{[en] Erasmus' selection of Publilio Siro's Sententiae and its influence on the florilegia of G. Maior and A. Rodrigues de Évora}

\begin{abstract}
Erasmus' edition of Publilio Siro's Sententiae (1514) constitutes a landmark in the literary fortune of this Roman mime and his work. This article studies the relationship between the sententiae of Publilio Siro selected by Erasmus and the Sententiae ueterum poetarum (1534) by G. Maior and the Sententiae et Exempla ex probatissimis scriptoribus collecta (1557) by A. Rodrigues de Évora. The analysis of these works will allow to offer a thematic classification of the Erasmian selection and to show to what extent these compilers have followed the Erasmian edition.
\end{abstract}

Key words: Publilius Syrus; sententiae; florilegia; Erasmus.

Sumario: 1. Introducción. 2. La selección de Erasmo. 3. Publilio Siro y su influencia en los florilegios latinos de G. Maior y A. Rodrigues de Évora. 4. La selección de Publilio Siro en los florilegios de G. Maior y de André Rodrigues de Évora: la influencia de la edición erasmiana. 5. Conclusiones. 6. Referencias bibliográficas. 6.1. Fuentes. 6.2. Estudios. Anexo. Sentencias de Publilio Siro editadas por Erasmo, agrupadas por temáticas y subtemas.

Cómo citar: Correa, S., «Las Sententiae de Publilio Siro seleccionadas por Erasmo y su influencia en los florilegios de G. Maior y A. Rodrigues de Évora», Cuad. Filol. Clásc. Estud. Lat. 38.1 (2018), 83-107.

$1 \quad$ Este trabajo se enmarca dentro del Proyecto de Investigación FFI2014-53859-P y la Red de Excelencia Europa Renascens. Biblioteca Digital de Humanismo y Tradición Clásica (España y Portugal) FFI2015-69200-REDT, financiados por el Gobierno de España.

2 Universidad de Las Palmas de Gran Canaria. Instituto Universitario de Análisis y Aplicaciones Textuales (IATEXT). francisco.bravo@ulpgc.es

3 Universidad de Las Palmas de Gran Canaria. Instituto Universitario de Análisis y Aplicaciones Textuales (IATEXT); gregorio.rodriguez@ulpgc.es. 


\section{Introducción}

Las Sententiae de Publilio Siro son una obra peculiar puesto que se nos han conservado y transmitido como una antología, lo que junto al carácter filosófico y didáctico de su contenido, las convierten en un claro ejemplo de obra de pensamiento fragmentario dentro del denominado género didáctico-ensayístico (García Berrio Huerta Calvo 1992, 222-224). Todo esto determina que su presencia en florilegios o compendios de carácter didáctico sea, como poco, una selección en segundo grado (Aldama - Muñoz 2005; Aldama - Muñoz 2009; Muñoz Jiménez 2004, 130; Muñoz Jiménez 2011).

Ya en la propia Roma se recogieron estas sentencias de Siro, tal y como lo ha estudiado Giancotti $(1967,278-338)$, probablemente para uso escolar, y durante la Edad Media a partir de la numerosas sentencias citadas por los dos Sénecas o Gelio se elabora un libro que será utilizado en la escuela, tal y como nos atestigua Jerónimo en Epist.107.8 (Herrero Llorente 1963, 265; Reeve 1983, 327; Vignes 2005, 193) y del que Giancotti encontró vestigios en bibliotecas francesas (Giancotti 1963, 57168).

La tradición manuscrita de Publilio, que ha sido ampliamente estudiada y catalogada por Giancotti (1963, 173-181), nos ha legado un conjunto de unos setecientos versos, a través de cinco fuentes manuscritas prinicpales (Reeve 1983: 327-329). La editio princeps ${ }^{4}$ se publica en Nápoles en 1475, aunque bajo la autoría de Séneca y sin recoger, por supuesto, la totalidad de las que hoy día atribuimos al mimo romano. Será Erasmo en Lovaina ${ }^{5}$ en 1514 el que elimine aquellas sentencias que de manera espúrea se habían vinculado al mimo y edite doscientas cuarenta y una sentencias que atribuye a Publilio Siro (Reeve 1983, 329).

La edición de Erasmo de las Sentencias de Publilio Siro forma parte de un opúsculo con varias obras. En éste encontramos textos tan variados como los Disthica Catonis, los Septem Sapiemtium Celebria Dicta o la Paraenesis Isocratis, traducida por Rodolfo Agrícola ${ }^{6}$. Sin embargo, el compendio que inicia la obra, los Disthica Catonis $^{7}$, será el que se imponga como denominación genérica del opúsculo en las numerosas ediciones posteriores, tanto del siglo XVI como del XVII ${ }^{8}$.

$4 \quad$ El término editio princeps para la edición de las Sententiae de Publilio Siro es controvertido ya que unos, como se ve en el cuerpo de nuestro trabajo, lo ajudican a la primera edición impresa de las sentencias, aun cuando estuviesen bajo la autoría de Séneca, y otros, como Giancotti, la reservan para la de Erasmo de 1514, dado que es ahí en donde el mimo romano ya aparece como el verdadero autor de los textos (Giancotti 1963, 13; Giancotti 1967, 339; Reeve 1983, 329).

5 Es muy frecuente encontrar en diferentes estudios que la primera edición se realizó en Londres y no en Lovaina, sin embargo Reeve (1983, 329 n. 17) afirma que "no other statement about the first printing of Erasmus' edition should be believed."

$6 \quad$ Una descripción completa de esta edición se puede consultar en Nijhoff-Kronenberg (1923, 199-200, $\left.\mathrm{n}^{\circ} 534\right)$.

7 Erasmo incluye en este opúsculo otras obras de pensamiento fragmentario de carácter didáctico-filosófico. Este compendio refuerza la inclusión de las Sententiae en el género didáctico-ensayístico, aunque en este caso recurriendo a la miscelánea, una variante del género de gran expansión en el Renacimiento (García Berrio - Huerta Calvo 1992, 222-224).

8 Amplios catálogos de las reediciones de las Sententiae de Publilio Syro pueden consultarse tanto en Vander Haeghen (1961 [= 1893]), como en el catálogo bibliográfico de la imprenta alemana de la Bibliotheksverbund Bayern https://opacplus.bib-bvb.de/TouchPoint_touchpoint/search.do?methodToCall=switchSearchPage\&SearchType $=2 \&$ emptyFields $=$ true [Consulta: $28 / 12 / 2017]$. 


\section{La selección de Erasmo}

La edición de Erasmo ${ }^{9}$ recoge 241 sentencias atribuidas al mimo latino a las que el humanista añade un breve comentario a la manera de accesus ad sententiam. La obra de Erasmo fue la principal difusora de las Sententiae de Publilio ${ }^{10}$. Estas sentencias tratan sobre temáticas diversas, que han recibido también varios intentos de clasificación. El tratamiento por temas de estas sentencias lo encontramos ya en el Giancotti (1967, 373-444). Según nuestros análisis, la selección de Erasmo puede responder a una tipología que, a excepción de siete sentencias (3\%), puede agruparse en cuatro grandes bloques ${ }^{11}$, a saber: temas genéricos sobre la visión del mundo, la sociedad y las relaciones humanas $(52 \%)$, virtudes $(12 \%)$, vicios $(23 \%)$ y estereotipos $(10 \%)$.

\begin{tabular}{|c|c|}
\hline TEMAS GENÉRICOS & SENTENCIAS \\
\hline El bien y el mal (hombres buenos y malvados) & 16 \\
\hline Fama/ elogio / gloria / buena reputación & 16 \\
\hline La muerte & 13 \\
\hline Amor & 12 \\
\hline Amistad /enemistad & 12 \\
\hline Miedos y peligros & 11 \\
\hline Culpa, pecado y castigo & 11 \\
\hline Pobreza y desgracia & 9 \\
\hline Fortuna & 6 \\
\hline Victoria & 5 \\
\hline Esclavitud & 3 \\
\hline Retraso, demora & 3 \\
\hline Destino & 2 \\
\hline Verdad & 2 \\
\hline Acuerdos y consejo & 2 \\
\hline Mal gobierno & 1 \\
\hline La razón & 1 \\
\hline Dolor & 1 \\
\hline Total & 126 \\
\hline
\end{tabular}

$9 \quad$ Citamos por la edición de Estrasburgo de 1515: Contenta in hoc opere sunt haec. Catonis praecepta moralia recognita atque interpretata ab Erasmo Roterodamo. Mimi Publiani. Septem sapientum illustres sententiae. Institutio hominis christiani versibus hexametris per Erasmum Roterodamum Isocratis paraenesis ad Demonicum Rudolpho Agricola interprete, recognita per Martinum Dorpium. Argentinae. Apud Ioannen Kloblouchium. MDXV (http://digital.onb.ac.at/OnbViewer/viewer.faces?doc=ABO_\%2BZ166256609 [Consulta: 28/12/2017]).

10 Las ediciones abarcan todo el siglo XVI, como puede comprobarse en los catálogos referenciados en las notas 5 y 7. Muchas de ellas pueden consultarse en las colecciones digitales de Europeana (www.europeana.eu) y en el catálogo digital de la Bayerische StaatsBibliothek (www.bsb_muenchen.de), [consulta 19/12/2017]. Además de esta cantidad de reediciones de la edición de Erasmo, se debe señalar la edición de H. Estienne (Ginebra 1569) en la que, por primera vez, se ordenan las sentencias por lemas (Vignes 2005, 193-194).

11 El anexo final recoge estas sentencias agrupadas por temas con una doble finalidad: evitar una cierta distracción en la lectura de la argumentación y análisis del trabajo, y ofrecer un listado de las sentencias de Publilio Siro seleccionadas por Erasmo, de manera que en otras investigaciones se puedan contejar con ediciones posteriores de otros humanistas como, por ejemplo, Escalígero que editó a Publilio Siro en 1598. 


\begin{tabular}{l|c}
\multicolumn{1}{c|}{ VIRTUDES } & SENTENCIAS \\
\hline Gratitud/ generosidad /beneficio & 14 \\
\hline Resignación /compasión/ misericordia & 8 \\
\hline Paciencia/ prudencia & 3 \\
\hline Valor/ audacia & 2 \\
\hline Perdón & 2 \\
\hline Total & 29
\end{tabular}

\begin{tabular}{l|c}
\multicolumn{1}{c|}{ VICIOS } & SENTENCIAS \\
\hline Avaricia & 12 \\
\hline Infamia / injuria & 11 \\
\hline Ira / cólera & 9 \\
\hline Envidia & 5 \\
\hline Prejuicios & 3 \\
\hline Molicie & 3 \\
\hline Desconfianza & 3 \\
\hline Desidia e indulgencia & 2 \\
\hline Ingratitud & 2 \\
\hline Adulación & 2 \\
\hline Hipocresía & 1 \\
\hline Soberbia & 1 \\
\hline Estupidez & 1 \\
\hline Discordia & 1 \\
\hline & Total \\
\hline
\end{tabular}

\begin{tabular}{|c|c|}
\hline ESTEREOTIPOS & SENTENCIAS \\
\hline La mujer & 8 \\
\hline La vejez & 4 \\
\hline Herencias y herederos & 4 \\
\hline Médicos y medicina & 2 \\
\hline El discípulo & 1 \\
\hline El borracho & 1 \\
\hline El tahur & 1 \\
\hline El charlatán & 1 \\
\hline El hombre & 1 \\
\hline Total & 23 \\
\hline
\end{tabular}

\section{Publilio Siro y su influencia en los florilegios latinos de G. Maior y A. Rodrigues de Évora}

Las Sententiae de Publilio tuvieron en el Renacimiento como receptores más inmediatos tanto a rétores y escritores como a estudiantes, que lo reutilizaban como autoridad ético-filosófica en la más pura tradición medieval (Pellegrin, 1978). Unos 
y otros tuvieron acceso no solo a las sucesivas reediciones de la compilación de Erasmo, sino también a diferentes florilegios ${ }^{12}$ en los que el mimo era uno de los autores extractados ${ }^{13}$ para dejarlo a disposición de la denominada res publica litteraria (López Poza 2000; Quondam 2003).

Sin embargo, no siempre encontramos la obra o el autor de la misma manera; de hecho podemos establecer una tipología sobre la presencia de las sentencias y de Publilio en los florilegios del XVI. La primera división se debe establecer entre aquellos florilegios en los que no aparecen ni el mimo ni sus sentencias, frente a otros en los que sí aparecen:

I. Florilegios en los que no aparecen Publilio Siro, ni sus Sententiae. Dada la fecha de la editio princeps de Erasmo, es lógico que el Viridarium illustrium poetarum (1507) de O. Mirándola no incluyese al mimo ${ }^{14}$. Este florilegio conoció tres ediciones más, aunque será su nuevo formato, los Illustrium poetarum flores (1538), el que obtenga mayor éxito y difusión ${ }^{15}$. Sin embargo, esta nueva versión del florilegio, que además va incorporando nuevas referencias en sucesivas ediciones, tampoco va a tener extractos de Publilio Siro. Otro florilegio relevante publicado también en la década de los treinta del siglo XVI que tampoco extractó a Publilio es el de R. Estienne, Sententiae et prouerbia ex poetis latinis (1534).

II. Florilegios en los que aparecen Publilio Siro y sus Sententiae.

En este apartado mostramos diferentes maneras en las que Publilio Siro y sus Sententiae son recogidos en los florilegios; tipologías, además, que pueden darse en un mismo florilegio.

II.1. Florilegios en los que las Sententiae de Publilio Siro aparecen como Sententiae Senecae. Dada la fecha de la edición de Erasmo, es evidente que la Polyanthea de D. Nano Mirabelio (1503) no podía incluir extractos del mimo, sin embargo sí que encontramos sus textos como Prouerbia Senecae. Esta asignación al filósofo cordobés de las sentencias permaneció en sucesivas ediciones de la Polyanthea a pesar de que, al mismo tiempo, el número de reimpresiones de la edición erasmiana también contribuía a la difusión de la obra del mimo romano; ni siquiera las ediciones de la Polyanthea de M. Cholinus (1585), ni la Polyanthea Noua (1604) de J. Lang, que revisan y aumentan los extractos del florilegio original de Nano Mirabelio, corrigen esta atribución (Artigas et alii 2014, 932-941; Moss 1996, 95-96; id. 205-206).

12 Aunque estas compilaciones de extractos recibieron numerosos y variados nombres como Manipulus, Polyanthea, Sententiae, Gnomai, Selecti Versus, Flores, Versus Sententiosi, Electa, Siluae, Collectanea, etc., nosotros utilizaremos el término "florilegio" como hiperónimo de toda esta variedad de denominaciones.

13 En el caso de Siro, más bien citado, ya que en los florilegios se reproducían las sentencias que habían sobrevivido.

14 Este florilegio estaba organizado por autores y los temas venían indicados en una Tabula alphabetica que remitía a las páginas y secciones.

15 Los Illustrium poetarum flores son un florilegio sistemático organizado en lemas o tituli ordenados alfabéticamente en los que se recogen sentencias sobre cada uno de los temas propuestos (Rodríguez Herrera 2010). Tuvieron, al menos, treinta y ocho ediciones entre 1538 y 1600 (Artigas et alii 2014,958) y es una reelaboración del Viridarium realizada por un escoliasta anónimo. 
II.2. Florilegios que extractan sentencias anónimas que realmente son de Publilio Siro. Las Sententiae prophanae ex diuersis scriptoribus in communem puerorum usum collectae per Leosthenem Coluandrum (Nuremberg, 1531), atribuidas a Leóstenes Coluandro, se encuentran dentro de este tipo. Se trata de un florilegio de sentencias ordenadas alfabéticamente sin ningún tipo de referencia a obra o autor. De hecho, y como el mismo título indica, son para uso escolar y parece un listado para ser aprendido de memoria. Aunque hubo otras ediciones de esta obra de manera independiente, en 1541 se publica junto al florilegio de Robert Estienne ${ }^{16}$, Sententiae et prouerbia ex poetis latinis $^{17}$.

La presencia de Publilio Siro en las Sententiae de L. Coluandro es escasa, apenas nueve extractos que tienen en común estar incluidos en la edición de Erasmo, que podría ser la fuente del compilador. Estas sentencias son las siguientes $^{18}$ :

Amare et sapere uix deo conceditur.

Consilio melius uincas quam iracundia.

Comes facundus in uia pro uehiculo est.

Ex uitio alterius sapiens emendat suum.

Feras quod laedit ut quod prodest perferas.

Inopi beneficium bis dat, qui dat celeriter.

Inimicum quamuis humilem docti est metuere.

Loco ignominiae est apud indignum dignitas.

Leuis est fortuna: cito reposcit quod dedit.

II.3. Florilegios que extractan sentencias anónimas falsamente atribuidas a Publilio Siro. La autoridad que emanaba de las sentencias de los autores clásicos, de un lado, y la transmisión del texto del mimo romano de manera fragmentaria y en formato antología, como ya hemos explicado, fomentó que un buen número de sentencias de carácter popular fuese atribuido a Publilio (Giancotti 1992, 9-10). Precisamente uno de los objetivos de Erasmo con su edición fue eliminar estas sentencias falsas (Herrero Llorente 1963, 263; Vignes 2005, 193). Aun así, estos textos espúreos se siguieron propagando a través de los florilegios y así lo encontramos tanto en las Sententiae ueterum poetarum, per locos communes digestae (Magdeburgo, 1534) de G. Maior, como, en mucha mayor medida, en las Sententiae et Exempla ex probatissimis

16 R. Estienne, Robertus Stephanus (1503-1559), impresor y humanista, fundó en 1526 una de las imprentas más relevantes del Renacimiento, en la que editó casi quinientas obras. Asimismo, como profundo conocedor del latín, el griego y el hebreo, publicó gran número de cuidadas ediciones anotadas de los autores latinos. También es el autor del más antiguo diccionario latino-francés, Dictionarium latino-gallicum (1513), y de un Thesaurus linguae latina (1532). Editó la Biblia y a él se debe su organización en versículos (Amstrong 1954).

17 R. Estienne publicó unas Sententiae et prouerbia ex omnibus Plauti et Terentii comoediis en 1530. Posteriormente en 1534 las publica ampliadas con otros extractos de poetas con el título de Sententiae et prouerbia ex poetis latinis. Como ya hemos dicho en 1541 aparecen editadas junto con las sentencias de L. Coluandro (Sententiae et prouerbia ex poetis latinis - Leosthenis Coluandri sententiae prophanas) y se reeditarán así al menos dos veces en Venecia en 1547 y 1552 (Artigas et alii 2014, 971-972). Nosotros citamos por la edición de Venecia de 1547.

18 Reproducimos las sentencias en el orden alfabético de la propia edición. 
scriptoribus collecta (Lyon, 1557) de André Rodrigues de Évora ${ }^{19}$, tal y como se ejemplifica en la siguiente tabla:

André Rodrigues de Évora,

Sententiae et Exempla ex probatissimis scriptoribus collecta

\begin{tabular}{|c|c|}
\hline Lema o titulus & Sententiae \\
\hline \multirow{2}{*}{ Ignauia } & Cum improbo uiro non facias iter. \\
\hline & Diues si desidiosus, pauper erit. \\
\hline Senectus & Frustra cum ad senectam uentum est, repetes adolescentiam. \\
\hline Lex & Improbus orator leges subuertit. \\
\hline Ingratitudo & Ingrato homini nihil peius terra creat. \\
\hline Inimicitia & Inimicis diffidens, numquam pateris incommodum. \\
\hline Mora et negligentia & Mora omnis odio est, facit sapientiam. \\
\hline Mulier & Mulier a muliere parum distat. \\
\hline Familia & Nulla pestis efficacior, quam familiaris inimicus. \\
\hline Infoelicitas, et tormentum & Oportet alios esse miseros, et alios foelices. \\
\hline Peregrinatio et absentia & Peregrinis succurrens paria obtinebis aliquando. \\
\hline Lex & Res fortis, lex est, si principem habeat. \\
\hline Inimicitia & Sermonem inimici numquam putes amicum \\
\hline Coniectura et suspicio & Suspicio, graue est hominibus malum. \\
\hline Secretum & Vel ne facias tegenda, uel solus facias. \\
\hline Vsura et mutuatio & Vsura liberos seruos faciunt \\
\hline
\end{tabular}

Lo más llamativo de estos extractos es que son realmente sentencias anónimas que parecen proceder de dichos populares. En algunos casos incluso parecen una traslación al latín de sentencias vernáculas como la conocida frase misógina "todas las mujeres son iguales", esto es: Mulier a muliere parum distat.

19 Ambos florilegios se estructuran en capítulos o lemas, pero, y este es un rasgo que vincula ambas compilaciones, no por orden alfabético, sino en una especie de agrupamiento onomasiológico, en el que se puede advertir la visión del mundo y de la sociedad de ambos. Por este motivo en la edición de los extractos seguimos el orden de aparición de los lemas o tituli bajo los que el compilador coloca cada sententia que como podrá advertirse no es un orden alfabético. 
II.4. Florilegios que atribuyen a Publilio Siro sentencias de otros autores. Este tipo de extractos se localizan, por ejemplo, en las Sententiae de André Rodrigues de Évora, tal y como puede apreciarse en la siguiente tabla:

André Rodrigues de Évora, Sententiae et Exempla ex probatissimis scriptoribus collecta

\begin{tabular}{l|l|l}
\hline Lema o titulus & \multicolumn{1}{|c}{ Sententiae } & \multicolumn{1}{|c}{ Autores } \\
\hline \multirow{4}{*}{ Inimicitia } & $\begin{array}{l}\text { Amicitia et inimicitia mortalium non sunt inmor- } \\
\text { tales, humana consilia reguntur temporibus mu- } \\
\text { tantur ratione utilitatis }\end{array}$ & $\begin{array}{l}\text { Erasmus Adagia } \\
3426\end{array}$ \\
\hline \multirow{4}{*}{ Matrimonium } & Argentum accepi dote, imperium uendendi. & $\begin{array}{l}\text { Erasmus Adagia } \\
4024 . \\
\text { Plaut.As.87. }\end{array}$ \\
\cline { 2 - 4 } & $\begin{array}{l}\text { Quae indutata est, ea in potestate est uiri: dum } \\
\text { modo morata recte ueniat, dotata est satis. }\end{array}$ & $\begin{array}{l}\text { Plaut.Aul.534- } \\
535\end{array}$ \\
\hline \multirow{3}{*}{ Mulier } & Mulieri imperare non concedit natura. & $\begin{array}{l}\text { Erasmus Adagia } \\
1481\end{array}$ \\
\cline { 2 - 4 } & Mulieris aemulatio totam turbat domum. & $\begin{array}{l}\text { Schottus, Adagia } \\
611\end{array}$ \\
\cline { 2 - 4 } & Thesaurus malorum est, mulier mala. & $\begin{array}{l}\text { Erasmus Adagia } \\
230\end{array}$ \\
\hline \multirow{2}{*}{$\begin{array}{l}\text { Alieno periculo } \\
\text { Sapere }\end{array}$} & Ex uitio alterius sapiens emendat suum. & $\begin{array}{l}\text { Erasmus } \\
\text { Adagia 1239 }\end{array}$ \\
\cline { 2 - 3 } & &
\end{tabular}

En el caso concreto de Rodrigues de Évora, este tipo de falsas atribuciones parece esconder un interés por extractar a Erasmo sin que pudiese ser percibida la presencia del humanistra neerlandés en esta edición de 1557, de manera que no se pudiera poner en riesgo la posición de su familia, que ya contaba con el estigma de proceder de cristianos nuevos (Lopes Andrade 2014, 321-323), si su florilegio parecía mostrar demasiadas simpatías por el pensamiento erasmiano. Esta hipótesis no se debe desdeñar ya que en las ediciones portuguesas (Lisboa 1554 y Coimbra 1569) publicadas bajo el título de Primera parte de las sentencias que hasta nuestros tiempos para edificacion de buenos costumbres, esta dispersos por Autores escriptas, en este tratado summariamente referidas en su propio estilo. Y traducidas en el nuestro comun. Conveniete licion a toda suerte y estado de gentes, se incluyeron 71 sentencias de Erasmo que fueron censuradas por la Inquisición (Lopes Andrade 2014, 328-329). Además en la edición de Lyon (1557) recurre a un reputado contrarreformista como Luis de Granada para el prólogo de sus Sententiae et Exempla. (Mack 2011, 269-273; Resina Rodrigues 1988, 770-772), de manera que su ortodoxia no pudiese ponerse en duda.

II.5. Florilegios que extractan sentencias de Publilio Siro.

Entre los florilegios del siglo XVI que extractan ya un considerable número de sentencias de Publilio Siro debemos destacar las ya citadas Sententiae ue- 
terum poetarum, per locos communes digestae de G. Maior y Sententiae et Exempla ex probatissimis scriptoribus collecta de André Rodrigues de Évora, a los que dedicaremos un análisis más pormenorizado en el siguiente apartado a partir de su vinculación con la edición de Erasmo.

\section{La selección de Publilio Siro en los florilegios de G. Maior y de André Rodrigues de Évora: la influencia de la edición erasmiana}

Las Sententiae ueterum poetarum de G. Maior ${ }^{20}$ (1534) son un florilegio de autores latinos clásicos y tardíos de carácter sistemático en el que los extractos se ordenan por lemas o tituli. Este florilegio tuvo un gran éxito en el Renacimiento, como lo atestiguan las cuarenta ediciones que se realizaron entre 1534 y $1600^{21}$ (Artigas et alii 2014, 967-68). Las sentencias de Publilio Siro presentes en este florilegio son 78 en total, distribuidas en 42 lemas, de las que 72 se encuentran en la edición de Eramo.

En la década de los cincuenta del siglo XVI André Eborense o André Rodrigues de Évora ${ }^{22}$ publica, en Lyon en $1557^{23}$, uno de los libros de lugares comunes de mayor difusión en los siglos XVI y XVII ${ }^{24}$, las Sententiae et Exempla ex probatissimis scriptoribus collecta. La obra está dividida en dos tomos que se corresponden con las Sententiae, el primero, y los Exempla, el segundo ${ }^{25}$, y que se ofrece a litterarum etiam morum atque pietatis studiosis ${ }^{26}$. Estas Sententiae son un florilegio de autores latinos clásicos y tardíos de carácter sistemático en el que los extractos se ordenan por lemas o tituli. Las sentencias de Publilio Siro presentes en este florilegio son 74 en total, distribuidos en 49 lemas, de las que 46 están recogidas en la edición de Erasmo. Estas sentencias recopiladas por Erasmo que también aparecen en los florilegios de G. Maior y de Rodrigues de Évora, así como el lema bajo el que cada compilador las agrupa se pueden consultar en la siguiente tabla:

20 G. Maior (1502-1574), teólogo protestante, fue rector de la Escuela de la ciudad de Mandeburgo entre 1529 y 1537, años en los que se publicaron sus Sententiae ueterum poetarum y la Quaestiones rhetoricae (1535). En 1545 se convirtió en profesor en la Facultad de Teología de Wittenberg en donde llegaría a ejercer como rector (Helmar 1987, 718).

21 Nosotros citamos por la edición de Amberes de 1541.

22 André Rodrigues da Veiga o de Évora, que firma sus obras como Andrea Eborensis Lusitanus, fue un rico comerciante afincado en Lisboa, hermano del médico y catedrático de Coimbra Tomás Rodrigues da Veiga y de Simón Rodriguez da Veiga, comerciante como André, pero residente en Bélgica (Costa Pimpao 1972, 387-401; Lopes Andrade 2014, 319-333). Es muy común confundirlo con el célebre humanista portugués André Rodrigues de Resende (Antonio 1788, 73a).

23 Sententia et Exempla ex probatissimis scriptoribus collecta per Andream Eborensem Lusitanum, Lugduni, apud Theobaldum Paganum, 1557. Costa Pimpao $(1972,395)$ considera que debió haber una edición anterior de Coimbra de 1554 , mientras que Juez Gálvez $(2009,268)$ la rechaza ya que no hay verdaderos indicios de su existencia, Artigas et alii $(2014,948$, n. 77) consideran que la edición de Coimbra de 1554 podría estar en el germen de la posterior de 1569 también de Coimbra, dado que por su estructura no es posible vincularla a la de Lyon de 1557.

24 Entre 1557 y 1635 se imprimen hasta dieciocho ediciones en diferentes ciudades europeas (Juez Gálvez 2009, 272-273).

25 Aragüés (1993, 252-265) trata las dos partes al mismo nivel; sin embargo como demuestran Codoñer Merino (2002), de un lado, y Juez Gálvez (2009), de otro, son dos obras muy diferentes que deben ser analizadas desde parámetros también diferentes. Para la descripción general de la obra véase Juez Gálvez (2009, 272-273).

26 Codoñer Merino $(2002,116)$ ha señalado ya el aire religioso de la compilación, así como los habituales tópicos sobre su utilidad presentes en el paratexto de las Sententiae et Exempla. 


\begin{tabular}{|c|c|c|}
\hline \multirow[t]{2}{*}{$\begin{array}{l}\text { Sentencias de Publilio Siro } \\
\text { recopiladas por Erasmo }{ }^{27}\end{array}$} & $\begin{array}{l}\text { Sentencias de Publilio Siro } \\
\text { recopiladas por G. Maior ( } 72 \text { ) }\end{array}$ & $\begin{array}{l}\text { Sentencias de Publi- } \\
\text { lio Siro recopiladas } \\
\text { por Rodrigues de } \\
\text { Évora (46) }\end{array}$ \\
\hline & Lema o titulus & Lema o titulus \\
\hline $\begin{array}{l}\text { 2. Ab alio expectes, alteri } \\
\text { quod feceris. }\end{array}$ & $\begin{array}{l}\text { Contra causidicos, et iniquos iu- } \\
\text { dices }\end{array}$ & $\begin{array}{l}\text { Aequalitas, similitu- } \\
\text { do, comparatio }\end{array}$ \\
\hline $\begin{array}{l}\text { 3. Animus uereri qui scit, scit } \\
\text { tuto aggredi. }\end{array}$ & Temeritas & Fortitudo et audacia \\
\hline $\begin{array}{l}\text { 4. Auxilia humilia firma con- } \\
\text { sensus facit. }\end{array}$ & Concordia & \\
\hline $\begin{array}{l}\text { 5. Amor, animi arbitrio sumi- } \\
\text { tur, non ponitur. }\end{array}$ & Luxus & \\
\hline $\begin{array}{l}\text { 6. Aut amat, aut odit mulier, } \\
\text { nihil est tertium. }\end{array}$ & Mulier & Mulier \\
\hline $\begin{array}{l}\text { 10. Amici uitia si feras, fa- } \\
\text { cias tua. }\end{array}$ & Aduersa interdum prosunt & Amicitia \\
\hline $\begin{array}{l}\text { 14. Auarus ipse miseriae cau- } \\
\text { sa est suae. }\end{array}$ & Auaritia & Cupiditas et auaritia \\
\hline $\begin{array}{l}\text { 22. Amare et sapere uix deo } \\
\text { conceditur. }\end{array}$ & Luxus & \\
\hline $\begin{array}{l}\text { 23. Auarus nisi cum moritur, } \\
\text { nil recte facit. }\end{array}$ & Auaritia & Cupiditas et auaritia \\
\hline $\begin{array}{l}\text { 26. Auaro quid mali optes; ni } \\
\text { ut uiuat diu. }\end{array}$ & Auaritia & Cupiditas et auaritia \\
\hline $\begin{array}{l}\text { 28. Alienum nobis, nostrum } \\
\text { plus aliis placet }\end{array}$ & Nemo sua sorte contentus & Inuidia \\
\hline $\begin{array}{l}\text { 32. Amicos res opimae pa- } \\
\text { rant, aduersae probant. }\end{array}$ & Aduersa interdum prosunt & Amicitia \\
\hline $\begin{array}{l}\text { 33. Ad poenitendum prope- } \\
\text { rat, cito qui iudicat. }\end{array}$ & Celeritas, praecipitatio & \\
\hline $\begin{array}{l}\text { 35. Arcum intensio frangit, } \\
\text { animum remissio. }\end{array}$ & Ocium ignauum & Seueritas \\
\hline $\begin{array}{l}\text { 36. Bis est gratum, quod } \\
\text { opus est, ultro si offeras. }\end{array}$ & $\begin{array}{l}\text { Contra causidicos, et iniquos iu- } \\
\text { dices }\end{array}$ & $\begin{array}{l}\text { Gratitudo et gratia- } \\
\text { rum actio }\end{array}$ \\
\hline
\end{tabular}

27 El número que precede a la sentencia se corresponde con el lugar que ocupa entre las 241 recopiladas por Erasmo. Hemos respetado la grafía de la edición a excepción de la 'u' intervocálica que hemos regularizado como ' $u$ ' ya que la propia edición vacila entre ' $u$ ' y 'v'. 


\begin{tabular}{|c|c|c|}
\hline $\begin{array}{l}\text { 37. Beneficium dare qui ne- } \\
\text { scit, iniuste petit. }\end{array}$ & Gratitudo & \\
\hline $\begin{array}{l}\text { 38. Bonum est, fugienda } \\
\text { aspicere alieno in malo. }\end{array}$ & Alieno periculo sapere & \\
\hline $\begin{array}{l}\text { 39. Beneficium accipere, li- } \\
\text { bertatem uendere est. }\end{array}$ & $\begin{array}{l}\text { Contra causidicos, et iniquos iu- } \\
\text { dices }\end{array}$ & \\
\hline $\begin{array}{l}\text { 42. Beneficia plura recipit, } \\
\text { qui scit reddere. }\end{array}$ & Gratitudo & \\
\hline $\begin{array}{l}\text { 43. Bis peccas, cum peccati } \\
\text { obsequium accommodas. }\end{array}$ & $\begin{array}{l}\text { Peccanti non praestandum obse- } \\
\text { quium }\end{array}$ & Obedientia \\
\hline $\begin{array}{l}\text { 44. Bonus animus numquam } \\
\text { erranti obsequium accomo- } \\
\text { dat. }\end{array}$ & $\begin{array}{l}\text { Peccanti non praestandum obse- } \\
\text { quium }\end{array}$ & Obedientia \\
\hline $\begin{array}{l}\text { 46. Beneficium dando acce- } \\
\text { pit, qui digno dedit. }\end{array}$ & $\begin{array}{l}\text { Contra causidicos, et iniquos iu- } \\
\text { dices }\end{array}$ & \\
\hline $\begin{array}{l}\text { 49. Beneficium se dedisse } \\
\text { qui dicit petit. }\end{array}$ & $\begin{array}{l}\text { Dantem obliuisci, accipientem } \\
\text { meminisse officii decet }\end{array}$ & Exprobatio \\
\hline $\begin{array}{l}\text { 51. Beneficium saepe dare, } \\
\text { docere est reddere. }\end{array}$ & $\begin{array}{l}\text { Contra causidicos, et iniquos iu- } \\
\text { dices }\end{array}$ & \\
\hline $\begin{array}{l}\text { 53. Bona opinio homini, tu- } \\
\text { tior pecunia est. }\end{array}$ & Bona fama & Fama \\
\hline $\begin{array}{l}55 . \text { Bis uincit, qui se uincit in } \\
\text { uictoria. }\end{array}$ & Moderatio & Victoria \\
\hline $\begin{array}{l}\text { 63. Bonis nocet, quisquis pe- } \\
\text { percerit malis. }\end{array}$ & Coercendi improbi & Prauitas \\
\hline $\begin{array}{l}\text { 65. Bonum apud uirum cito } \\
\text { moritur iracundia }\end{array}$ & Ira & Ira \\
\hline $\begin{array}{l}\text { 69. Beneficium dignis, ubi } \\
\text { das, omnes obligas. }\end{array}$ & $\begin{array}{l}\text { Contra causidicos, et iniquos iu- } \\
\text { dices }\end{array}$ & \\
\hline $\begin{array}{l}\text { 75. Cum inimico nemo in } \\
\text { gratiam cito redit }\end{array}$ & Inimicitia & Inimicitia \\
\hline $\begin{array}{l}\text { 78. Cito ignominia fit, super- } \\
\text { bi gloria. }\end{array}$ & Arrogantia, iactantia & Superbia \\
\hline $\begin{array}{l}\text { 79. Consilio melius uincas } \\
\text { quam iracundia. }\end{array}$ & Ira & Ira \\
\hline $\begin{array}{l}\text { 80. Cuiuis dolori remedium } \\
\text { est patientia. }\end{array}$ & Patientia & Patientia \\
\hline $\begin{array}{l}\text { 83. Comes facundus, in uia } \\
\text { pro uehiculo est. }\end{array}$ & Eloquentia et eius uis & \\
\hline
\end{tabular}




\begin{tabular}{|c|c|c|}
\hline $\begin{array}{l}\text { 87. Discipulus est prioris, } \\
\text { posterior dies. }\end{array}$ & Experientia & \\
\hline $\begin{array}{l}\text { 90. Dixeris maledicta cunc- } \\
\text { ta, cum ingratum hominem } \\
\text { dixeris. }\end{array}$ & Ingratitudo & Ingratitudo \\
\hline $\begin{array}{l}\text { 95. Discordia fit charior con- } \\
\text { cordia }\end{array}$ & Concordia & \\
\hline $\begin{array}{l}\text { 97. Difficilem oportet aurem } \\
\text { habere ad crimina. }\end{array}$ & Obtretactio & \\
\hline $\begin{array}{l}\text { 99. Damnum appellandum } \\
\text { est, cum mala fama lucrum. }\end{array}$ & Lucrum praeda & \\
\hline $\begin{array}{l}101 . \text { Dies quod donat timeas } \\
\text { cito raptum uenit. }\end{array}$ & Fortuna et eius inconstantia & \\
\hline $\begin{array}{l}\text { 102. Feras non culpes, quod } \\
\text { mutari non potest. }\end{array}$ & Patientia & Patientia \\
\hline $\begin{array}{l}\text { 104. Furor fit laesa saepius } \\
\text { patientia }\end{array}$ & Patientia & Patientia \\
\hline $\begin{array}{l}\text { 105. Fidem qui perdit, nil po- } \\
\text { test ultra perdere. }\end{array}$ & Seruanda fides, praesertim & Veritas et affirmatio \\
\hline $\begin{array}{l}\text { 111. Frustra, cum ad senec- } \\
\text { tam uentum est, repetes ado- } \\
\text { lescentiam. }\end{array}$ & De aetate hominum & Senectus \\
\hline $\begin{array}{l}\text { 116. Fortuna uitrea est; quae } \\
\text { cum splendet frangitur. }\end{array}$ & Fortuna et eius inconstantia & Foelicitas \\
\hline $\begin{array}{l}127 . \text { Grauissimum est impe- } \\
\text { rium consuetudinis. }\end{array}$ & Mores, consuetudo & Vsus et consuetudo \\
\hline $\begin{array}{l}\text { 130. Homo extra corpus est } \\
\text { suum, cum irascitur. }\end{array}$ & Ira & Ira \\
\hline $\begin{array}{l}\text { 142. Honestus rumor alterum } \\
\text { est patrimonium. }\end{array}$ & Bona fama & Fama \\
\hline $\begin{array}{l}\text { 154. In nullum auarus bonus } \\
\text { est, in se pessimus. }\end{array}$ & Auaritia & Cupiditas et auaritia \\
\hline $\begin{array}{l}\text { 163. Inopiae parua desunt, } \\
\text { auaritiae omnia. }\end{array}$ & Auaritia & Cupiditas et auaritia \\
\hline $\begin{array}{l}\text { 164. Ita amicum habeas, pos- } \\
\text { se ut fieri inimicum putes. }\end{array}$ & Aduersa interdum prosunt & \\
\hline $\begin{array}{l}\text { 169. Iniuriarum remedium } \\
\text { est obliuio. }\end{array}$ & Obliuio & Iniuria et contemptus \\
\hline
\end{tabular}




\begin{tabular}{|c|c|c|}
\hline $\begin{array}{l}\text { 170. Iracundiam qui uincit, } \\
\text { hostem superat maximum }\end{array}$ & Ira & Ira \\
\hline $\begin{array}{l}\text { 179. Ita crede amico, ne sit } \\
\text { inimico locus. }\end{array}$ & Aduersa interdum prosunt & Amicitia \\
\hline $\begin{array}{l}\text { 181. Inprobe Neptunum ac- } \\
\text { cusat, qui iterum naufragium } \\
\text { facit. }\end{array}$ & Semel laesus timet, et cauet & Mare et nauigatio \\
\hline $\begin{array}{l}185 . \text { Leuis est fortuna, cito } \\
\text { reposcit, quae dedit. }\end{array}$ & Fortuna et eius inconstantia & \\
\hline $\begin{array}{l}\text { 187. Lucrum sine damno al- } \\
\text { terius fieri non potest. }\end{array}$ & Lucrum praeda & \\
\hline $\begin{array}{l}\text { 190. Luxuriae desunt multa, } \\
\text { auaritiae omnia. }\end{array}$ & Auaritia & \\
\hline $\begin{array}{l}\text { 193. Mora omnis odio est, } \\
\text { sed facit sapientiam. }\end{array}$ & Mora & Mora et negligentia \\
\hline $\begin{array}{l}\text { 197. Miserrima est fortuna, } \\
\text { quae inimico caret. }\end{array}$ & Inuidia, liuor, odium & $\begin{array}{l}\text { Infoelicitas et tormen- } \\
\text { tum }\end{array}$ \\
\hline $\begin{array}{l}\text { 206. Malo in consilio foemi- } \\
\text { nae uincunt uiros. }\end{array}$ & Mulier bona & \\
\hline $\begin{array}{l}\text { 208. Magno cum periculo cus- } \\
\text { toditur, quod multis placet. }\end{array}$ & Forma & \\
\hline $\begin{array}{l}\text { 209. Mala est medicina, ubi } \\
\text { aliquid naturae perit. }\end{array}$ & Medicina & Medicina \\
\hline $\begin{array}{l}\text { 220. Multos timere debet, } \\
\text { quem multi timent. }\end{array}$ & Tyrannis & Tyrannis \\
\hline $\begin{array}{l}\text { 221. Male imperando sum- } \\
\text { mum imperium amittittur. }\end{array}$ & Tyrannis & \\
\hline $\begin{array}{l}\text { 228. Nescis quid optes, aut } \\
\text { quid fugias, ita ludit dies. }\end{array}$ & $\begin{array}{l}\text { Misera hominum uita et omnia } \\
\text { caduca ac fragilia }\end{array}$ & $\begin{array}{l}\text { Mutatio et inconstan- } \\
\text { tia }\end{array}$ \\
\hline $\begin{array}{l}\text { 229. Nunquam periculum } \\
\text { sine periculo uincitur. }\end{array}$ & Audacia & Periculum \\
\hline $\begin{array}{l}\text { 233. Nimium altercando ue- } \\
\text { ritas amittitur. }\end{array}$ & Contentio & Litigium \\
\hline $\begin{array}{l}\text { 238. Stultum est timere, quod } \\
\text { uitari non potest. }\end{array}$ & Metus, Timor & Timor et turbatio \\
\hline $\begin{array}{l}240 . \text { Veterem ferendo, iniuri- } \\
\text { am inuitas nouam. }\end{array}$ & Coercendi improbi & Inuria et contemptus \\
\hline $\begin{array}{l}\text { 241. Tam deest auaro quod } \\
\text { habet quam quod non habet. }\end{array}$ & Auaritia & \\
\hline
\end{tabular}


Del análisis de estas sentencias podemos establecer que la dependencia de estos florilegios de la edición de Erasmo es diferente en uno y en otro. Mientras que en el florilegio de G. Maior la vinculación entre sus extractos y los de Erasmo se eleva hasta el 97\%, pues de las 78 sentencias atribuidas a Publilio 72 están recogidas en la edición erasmiana; en el caso de Rodrigues de Évora esta relación se reduce al 64\%, lo que presupone que sus fuentes para los extractos de mimo romano fueron más allá de la edición de Erasmo.

En cuanto al vínculo entre ambos florilegios destacamos que las 46 sentencias recogidas por Rodrigues de Évora también aparecen en el florilegio de Maior ${ }^{28}$ y que 27 de ellas comparten un mismo lema o $\operatorname{similar}^{29}$. En cuanto a la relación entre los lemas y los cuatro grandes grupos temáticos que establecimos al principio de este trabajo en la selección de Erasmo, podemos concluir que tanto en G. Maior como en Rodrigues de Évora predominan los extractos de temas generales, aunque esto es normal dado que este apartado agrupa la mayor cantidad de extractos, seguidos de aquellos que denuncian los vicios, que casi duplican a las virtudes, mientras que los estereotipos tienen una escasa presencia:

Temática de los extractos de Publilio en los florilegios

\begin{tabular}{l|c|c|c|c}
\hline & Generales & Virtudes & Vicios & Miscelánea \\
\hline G. Maior & 31 & 13 & 21 & 6 \\
\hline Rodrigues de Évora & 20 & 8 & 15 & 3
\end{tabular}

La selección de Rodrigues de Évora, además, esconde en cierta medida la vinculación de su florilegio con Erasmo, no solo en lo que al mimo romano se refiere, sino también en la atribución a Publilio de adagios erasmianos. Evidencia la selección, por tanto, un intento de esconder la figura del Erasmo como fuente de su obra.

\section{Conclusiones}

En primer lugar debemos destacar que Publilio Siro y sus Sententiae no han ido siempre de la mano en los florilegios latinos del siglo XVI, pues, a pesar de la edición de Erasmo en 1514, podemos encontrar que las sentencias son atribuidas a Séneca o se transmiten de manera anónima y que otras son falsamente atribuidas al mimo. En aquellos florilegios en los que Sententiae y autor sí van de la mano, puede advertirse que en un primer momento la influencia de la edición de Erasmo es muy alta y así lo atestigua el florilegio de G. Maior (1534), pero que a medida que avanza el siglo esta parece reducirse. Así, el florilegio de A. Rodrigues de Évora (1557) toma de la edición erasmiana sólo el 64\% de las sentencias. La selección de A. Rodrigues de Évora es menos rigurosa que la de G. Maior, pues a las siete sentencias, en su mayoría procedentes de los Adagia de Erasmo, hay que añadir otras dieciséis de carácter

28 Esta vinculación entre ambos florilegios ya ha sido establecida a propósito de los extractos de Ovidio presentes en las Sententiae et Exempla de A. Rodrigues de Évora (Rodríguez Herrera 2016; Rodríguez Herrera 2017).

29 Situar las sentencias bajo un lema u otro es una muestra de la intención del compilador y de la manipulación de los textos de los autores clásicos (Muñoz Jiménez 2004, 128). 
popular que atribuye falsamente a Publilio Siro, de manera que su florilegio, a pesar del esfuerzo hecho por Erasmo por expurgar la obra conservada del mimo romano, supone un paso atrás en la autoría de las Sententiae de Publilio Siro; un paso atrás aumentado por el hecho de que este florilegio gozó de una gran difusión e influencia en los siglos XVI y XVII.

Por último, debemos señalar que las sentencias recogidas en los florilegios abarcan los grandes grupos temáticos establecidos en la selección de Erasmo, aunque en ambos predominan los temas de carácter general sobre la concepción del mundo, la sociedad y las relaciones humanas, así como los que critican los vicios.

\section{Referencias bibliográficas}

\subsection{Fuentes}

Erasmo de Roterdam, D. (1515), Contenta in hoc opere sunt haec. Catonis praecepta moralia recognita atque interpretata ab Erasmo Roterodamo. Mimi Publiani. Septem sapientum illustres sententiae. Institutio hominis christiani versibus hexametris per Erasmum Roterodamum Isocratis paraenesis ad Demonicum Rudolpho Agricola interprete, recognita per Martinum Dorpium. Argentinae, apud Ioannen Kloblouchium.

Estienne, R. (1547), Sententiae et prouerbia ex poetis latinis - His adiecimus Leosthenis Coluandri sententias prophanas. Venetiis.

Herrero Llorente, V. J. (1963), Publilio Siro. Sentencias (suplementos de «Estudios Clásicos»), Madrid, Sociedad Española de Estudios Clásicos.

Maior, G. (1541), Sententiae ueterum poetarum, per locos communes digestae. Collectore Georgio Maiore. Antuerpiae, per Antonium Dumaeum [=Impressum Madeburgi, apud Michaelem Lotherum, 1534].

Meyer, W. (1880), Publilii Syri Mimi Sententiae, Leipzig, Teubner (PHI Latin Texts < http:// latin.packhum.org/author/622> [Consulta: 21/02/2017]).

Rodrigues de Évora, A. (1557), Sententiae et Exempla ex probatissimis scriptoribus collecta et per locos communes digesta per Andream Eborensem Lusitanum, Ludugni, apud Theobaldum Paganum.

Woelfflin, E. (1869), Publilii Syri Sententiae, Leipzig, Teubner.

Bibliotheksverbund Bayern (https://opacplus.bib-bvb.de/TouchPoint_touchpoint/search. do?methodToCall=switchSearchPage $\&$ SearchType $=2 \&$ emptyFields $=$ true $\quad$ [Consulta: 28/12/2017]).

\subsection{Estudios}

Aldama Roy, A. M ${ }^{a}$-Muñoz Jiménez, Mª J. (2005), "Los florilegios”, en B. Antón et alii, Antiquae lectiones, Madrid, Cátedra, 217-223.

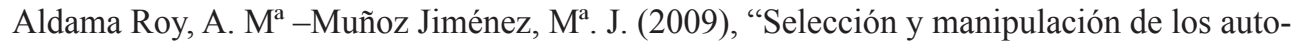
res clásicos en los florilegios latinos", en $\mathrm{M}^{\mathrm{a}}$. D. García de Paso Carrasco - G. Rodríguez Herrera (eds.), Selección, manipulación y uso metaliterario de los autores clásicos, Zaragoza, Pórtico, 99-123. 
Amstrong, E. (1954), Robert Estienne, Royal Printer: An Historical Study of the Elder Stephanus, Cambridge, Cambridge University Press.

Antonio, N. (1788), Bibliotheca Hispana Noua siue Hispanorum Scriptorum qui ab anno MD ad MDCXXXIV floruere notitia, Madrid.

Aragüés, J. (1993), “Colecciones de Exempla y oratoria: la labor del compilador”, Humanismo y pervivencia del mundo clásico 1.1, Cádiz, 252-265.

Artigas, E. et alii (2014), "De floribus florilegiisque barcinonensibus", M". T. Callejas Berdonés et alii, Manipulus Studiorum en recuerdo de la profesora Ana María Aldama Roy, Madrid, 921-1056.

Codoñer Merino, C. (2002), “La educación hispánica en el siglo XVI. La compilación de Sententiae de André Rodrigues Eborense", Cataldo e André de Resende. Congreso Internacional do Humanismo Português, Lisboa, 111-121.

Costa Pimpão, A. J. da (1972), "André Eborense e o seu livro de Sentenças e Exemplos", Escritos diversos. Coimbra, 387-401.

García Berrio, A., Huerta Calvo, J. (1992), Los géneros literarios: sistema e historia, Madrid, Cátedra.

Giancotti, F. (1963), Ricerche sulla tradizioine manoscrita delle sentenze di Publilio Siro, Messina-Firenze, Biblioteca di Cultura Contemporanea.

Giancotti, F. (1967), Mimo e Gnome. Studio su Decimo Laberio e Publilio Siro, Messina-Firenze, Casa Editrice G. D’Anna.

Giancotti, F. (1992), "Le Sententiae di Publilio Siro e Seneca”, La langue latine, langue de la philosophie. Actes du colloque de Rome, Roma, École Française de Rome, 9-38.

Helmar, J. (1987), "Major, Georg", Neue Deutsche Biographie 15, p. 718. < http://www. deutsche-biographie.de/pnd116689781.html> [Consulta: 07/03/2016].

Juez Gálvez, F. J. (2009), “André Rodrigues de Évora, un maruliano portugués del siglo XVI", Colloquia Maruliana 18, 267-285.

Lopes Andrade, A. M. (2014), O Cato Minor de Diogo Pires e a Poesia didáctica do século $X V I$, Lisboa, Impresa Nacional - Casa da Moneda.

López Poza, S. (2000), "Polianteas y otros repertorios de utilidad para la edición de textos en el Siglo de Oro", La Perinola 4, 191-207.

Mack, P. (2011), A History of Renaissance Rhetoric 1380-1620, Oxford, Oxford University Press.

Moss, A. (1996), Printed Commonplace-Books and the Structuring of Renaissance Thought, Oxford, Clarendon Press.

Muñoz Jiménez, Ma. J. (2004), "La edición de florilegios como edición especial”, Exemplaria clasica $8,123-133$.

Muñoz Jiménez, Ma . J. (2011), "Formas de coexistencia de los autores y obras en los florilegios latinos", en $\mathrm{M}^{\mathrm{a}}$. J. Muñoz Jiménez (ed.), El florilegio: espacio de encuentro de los autores antiguos y medievales. Porto, Fédération Internationale del Instituts d'Études Médiévales, 135-153.

Nijhoff, W., Kronenberg, M. E. (1923-1971), Nederlandsche Bibliographie van 1500 tot 1540, s'Gravenhage, ${ }^{\circ} 534,3$ v, 199-200.

Pellegrin, E. (1978), "Notes sur un commentaire médiéval des Sententiae de Publius Syrus", Revue d'histoire des textes 6, 305-322.

Quondam, A. (2003), “Strumenti dell'officina classicistica: Polyanthea \& Co.”, Modern Philology 101/2, 316-335.

Reeve, M. D. (1983), "Publilius", en L. D. Reynolds, Texts and Transmission. A Survey of the Latin Classics, Oxford, Oxford University Press, 327-329. 
Resina Rodrigues, Ma . I. (1988), Fray Luis de Granada y la literatura de espiritualidad en Portugal (1554-1632), Madrid, Universidad Pontificia de Salamanca - Fundación Universitaria Española.

Rodríguez Herrera, G. (2010), "Propercio en el Viridarium Illustrium Poetarun de Octaviano Mirándola: origen e influencias”, Cuadernos de Filología Clásica. Estudios Latinos 30/2, 295-320.

Rodríguez Herrera, G. (2016), "La selección de los elegiacos latinos en las Sententiae et Exemplum (1557) de André Rodrigues de Évora", Habis 47, 269-284.

Rodríguez Herrera, G. (2017), "Ovidio en las Sententiae et Exempla (1557) de André Rodrigues de Évora: Fastos, Tristes y Pónticas", Actas do Congresso Internacional O Humanismo Português e Europeu no 5. ${ }^{\circ}$ centenário do Cicero Lusitanus, Jerónimo Osório (1515-1580). Lisboa-Coimbra.

Vander Haeghen, F., Bibliotheca Erasmiana. Répertoire des oeuvres d'Erasme, Nieuwkoop, 1961 (= Gent, 1893)

Vignes, J. (2005), "Pour une gnomologie: Enquête sur la succès de la littérature gnomique à la Renaissance", Seizième Siècle 1, 175-211. 


\section{ANEXO}

Sentencias de Publilio Siro editadas por Erasmo, agrupadas por Temáticas y subTEMAS

I.- TEMAS DE CARÁCTER GENERAL SOBRE LA CONCEPCIÓN DE EL MUNDO, LA SOCIEDAD Y LAS RELACIONES HUMANAS:

\section{El bien y el mal (hombres buenos y malvados)}

2. Ab alio expectes, alteri quod feceris.

54. Bonum tametsi supprimitur, non extinguitur.

58. Bene dormit, qui non sentit, quam male dormiat.

59. Bonorum crimen est officiosus miser.

61. Bene cogitata si excidunt, non occidunt.

64. Bono iustitiae proxima est seueritas.

84. Cito inproborum laeta ad perniciem cadunt.

124. Grauius est malum omne, quod sub aspectu latet.

125. Grauius nocet, quodcunque inexpertum accidit.

159. Ingenuitas non recipit contumeliam.

198. Malus est uocandus, qui sua causa est bonus.

199. Malus ubi bonum se simulat, tunc est pessimus.

210. Malae naturae nunquam doctrina indigent.

218. Malefacere qui uult, nusquam non causam inuenit.

236. Ridiculum est odio nocentis perdere innocentiam.

239. Timidus uocat se cautum, parcum sordidus.

\section{Fama / elogio/ gloria / buena reputación}

53. Bona opinio homini, tutior pecunia est.

60. Bona fama in tenebris proprium splendorem obtinet.

99. Damnum appellandum est, cum mala fama lucrum.

105. Fidem qui perdit, nil potest ultra perdere.

108. Fidem nemo unquam perdit, nisi qui non habet.

119. Frugalitas miseria est rumoris boni.

129. Heu quam difficile est gloriae custodia.

133. Est honesta turpitudo pro bona causa mori.

142. Honestus rumor alterum est patrimonium.

153. Ingenuitatem laedis, cum indignum rogas.

182. Loco ignominiae est apud indignum dignitas.

183. Laus ubi noua oritur, etiam uetus admittitur.

185. Leuis est fortuna; cito reposcit, quae dedit.

188. Lasciuia et laus nunquam habent concordiam.

202. Male geritur quicquid geritur fortunae fide.

224. Nihil agere semper infoelici est optimum.

\section{La muerte}

41. Bis enim mori est, alterius arbitrio mori.

45. Bona mors est homini, uitae quae extinguit mala.

57. Bis interimitur, qui suis armis perit.

74. Cuius mortem amici expectant, uitam oderunt. 
85. Crimen relinquit uitae qui mortem appetit.

98. Dum uita grata est, mortis conditio optima est.

129. Heu quam est timendus, qui mori tutum putat.

140. Homo toties moritur, quoties amittit suos.

186. Lux uniuersi est, quae iubet nasci ac mori.

195. Mori est foelicis antequam mortem inuocet.

201. Mori necesse est, sed non quoties uolueris.

203. Mortuo qui mittit munus, nil dat illi sibi adimit.

231. Nusquam melius morimur homines, quam ubi libenter uiximus.

\section{Amor}

5. Amor, animi arbitrio sumitur, non ponitur.

8. Ames parentem si aequus est, si alter feras.

13. Amans iratus multa mentitur sibi.

15. Amans quid cupiat scit, quid sapiat non uidet.

16. Amans quod suspicatur uigilans, somniat.

18. Amor extorqueri non potest, elabi potest.

19. Ab amante lachrimis redimas iracundiam.

22. Amare et sapere uix deo conceditur.

29. Amare iuueni fructus est, crimen seni.

31. Amoris uulnus idem qui sanat facit.

47. Blanditia, non imperio fit dulcis Venus.

166. In amore mendax semper iracundia est.

\section{Amistad / enemistad}

10. Amici uitia si feras, facias tua.

32. Amicos res optimae parant, aduersae probant.

75. Cum inimico nemo in gratiam cito redit.

91. De inimico ne loquares malum, si cogites.

126. Grauior inimicus qui latet sub pectore.

150. Inimicum ulcisci, uitam accipere est alteram.

164. Ita amicum habeas, posse ut fieri inimicum putes.

168. Iratum breuiter uites, inimicum diu.

173. Inimicum quamuis humilem docti est metuere.

179. Ita crede amico, ne sit inimico locus.

184. Laeso doloris remedium inimici dolor.

197. Miserrima est fortuna, quae inimico caret.

\section{Miedos y peligros}

3. Animus uereri qui scit, scit tuto aggredi.

76. Citius uenit periculum, cum contemnitur.

101. Dies quod donat timeas cito raptum uenit.

200. Metus cum uenit, rarum habet somnus locum.

208. Magno cum periculo custoditur, quod multis placet.

211. Miseriam nescire, est sine periculo uiuere.

220. Multos timere debet, quem multi timent.

227. Non cito ruina perit uir, qui ruinam timet.

229. Nunquam periculum sine periculo uincitur. 
234. Quotidie damnatur, qui semper timet.

238. Stultum est timere, quod uitari non potest.

\section{Culpa, pecado y castigo}

43. Bis peccas, cum peccati obsequium accommodas.

48. Bonus animus numquam erranti obsequium accomodat.

62. Bene perdit nummos, iudici cum dat nocens.

81. Cum uitia prosunt, peccat qui recte facit.

122. Grauis animi poena est, quem post factum poenitet.

149. Inferior horret, quicquid peccat superior.

157. Inuitat culpam, qui peccatum praeterit.

175. Iudex damnatur, cum nocens absoluitur.

178. Illo nocens se damnat, quo peccat die.

181. Inprobe Neptunum accusat, qui iterum naufragium facit.

189. Legem nocens ueretur, fortunam innocens.

\section{Pobreza y desgracia}

38. Bonum est, fugienda aspicere alieno in malo.

70. Crudelis in re aduersa est obiurgatio.

88. Damnare est obiurgare, cum auxilio est opus.

136. Hominem experiri multa paupertas iubet.

137. Heu dolor, quam miser est qui in tormento uocem non habet.

156. Instructa inopia est in diuitiis cupiditas.

162. In miseri uitia nulla contumelia est.

171. In malis sperare bene, nisi innocens nemo solet.

174. In calamitoso risus etiam iniuria est.

\section{Fortuna}

109. Fortuna obesse nulli contenta est semel.

115. Fortuna nulli plusquam consilium ualet.

116. Fortuna vitrea est; quae cum splendet frangitur.

118. Facit gratum fortuna, quam nemo uidet.

143. Homo nescit si dolore fortunam inuenit.

230. Nulla tam bona est fortuna, de qua nil possis queri.

\section{Victoria}

55. Bis uincit, qui se uincit in uictoria.

79. Consilio melius uincas quam iracundia.

89. Diu adparandum est bellum, ut uincas celerius.

103. Futura pugnant, ut se superari sinant.

212. Male uiuunt, qui se semper uicturos putant.

\section{Esclavitud}

11. Aliena homini ingenuo, acerba est seruitus.

144. Honeste seruit qui succumbit tempori.

204. Minus est quam seruus, dominus, qui seruos timet. 


\section{Retraso, demora}

92. Deliberare utilia, mora est tutissima.

96. Deliberandum est diu, quod statuendum est semel.

123. Grauis animus dubiam non habet sententiam.

\section{Destino}

28. Nescis quid optes, aut quid fugias, ita ludit dies.

141. Homo semper in se aliud fert, in alterum aliud cogitat.

\section{Verdad}

190. Iucundum nihil est, nisi quod reficit ueritas.

233. Nimium altercando ueritas amittitur.

\section{Acuerdos y consejo}

4. Auxilia humilia firma consensus facit.

223. Malum consilium est quod mutari non potest.

\section{Mal gobierno}

221. Male imperando summum imperium amittitur.

\section{La razón}

225. Nil peccent oculi, si animus oculis imperet.

\section{Dolor}

93. Dolor decrescit, ubi quo crescat non habet.

\section{VIRTUDES :}

\section{Gratitud / generosidad / beneficio}

36. Bis est gratum, quod opus est, ultro si offeras.

37. Beneficium dare qui nescit, iniuste petit.

39. Beneficium accipere, libertatem uendere est.

40. Bona nemini hora est, ut non alicui sit mala.

42. Beneficia plura recipit, qui scit reddere.

46. Beneficium dando accepit, qui digno dedit.

49. Beneficium se dedisse qui dicit, petit.

51. Beneficium saepe dare, docere est reddere.

56. Benignus etiam dandi causam cogitat.

69. Beneficium dignis, ubi das, omnes obligas.

72. Cui semper dederis, ubi neges, rapere imperas.

134. Habet in aduersis auxilia, qui in secundis commodat.

155. Inopi beneficium bis dat, qui dat celeriter.

237. Pars beneficii est, quod petitur, si bene neges.

\section{Resignación / compasión/ misericordia}

67. Bona comparat praesidia misericordia.

102. Feras non culpes, quod mutari non potest.

114. Fer difficilia, ut facilia leuius feras.

117. Feras quod laedit, ut quod prodest perferas. 
132. Homo, qui in homine calamitoso est misericors, meminit sui.

194. Mala causa est quae requirit misericordiam.

196. Miserum est tacere cogi, quod cupias loqui.

240. Veterem ferendo iniuriam, inuitas nouam.

\section{Paciencia / prudencia}

80. Cuiuis dolori remedium est patientia (Meyer sapientia).

104. Furor fit laesa saepius patientia.

193. Mora omnis odio est, sed facit sapientiam.

\section{Valor / audacia}

100. Ducis in consilio, posita est uirtus militum.

177. In rebus dubiis plurimi est audacia.

\section{Perdón}

63. Bonis nocet, quisquis pepercerit malis.

176. Ignoscere hominum, ubi pudet cui ignoscitur.

III.- VICIOS:

\section{Avaricia}

14. Auarus ipse miseriae causa est suae.

21. Auarum facile capias, ubi non sis idem.

23. Auarus nisi cum moritur, nil recte facit.

25. Auarus damno potius, quam sapiens dolet.

26. Auaro quid mali optes; ni ut uiuat diu.

154. In nullum auarus bonus est, in se pessimus.

163. Inopiae parua desunt, auaritiae omnia.

187. Lucrum sine damno alterius fieri non potest.

190. Luxuriae desunt multa, auaritiae omnia.

191. Malignos fieri, maxime ingrati docent.

232. Negandi causa auaro nunquam deficit.

241. Tam deest auaro quod habet, quam quod non habet.

\section{Infamia / injuria}

17. Ad calamitatem quilibet rumor ualet.

97. Difficilem oportet aurem habere ad crimina.

112. Falsum maledictum, maleuolum mendacium est.

128. Graue crimen, etiam cum dictum est leuiter, nocet.

135. Heu quam miserum est ab illo laedi, de quo non possis quaeri.

148. Habent locum maledicti crebrae nuptiae.

169. Iniuriarum remedium est obliuio.

192. Multis minatur, qui uni facit iniuriam.

213. Maledictum interpretando, facies acrius.

216. Mutat se bonitas, cum irrites iniuria.

219. Maleuolus semper sua natura uescitur.

\section{Ira / cólera}

44. Bonus animus laesus, grauius multo irascitur. 
65. Bonum apud uirum cito moritur iracundia.

66. Bona turpitudo est quae periculum indicat.

104. Furor fit laesa saepius patientia.

110. Fulmen est, ubi cum potestate habitat iracundia.

121. Grauissima est probi hominis iracundia.

130. Homo extra corpus est suum, cum irascitur.

170. Iracundiam qui uincit, hostem superat maximum.

180. Iratus etiam facinus consilium putat.

\section{Envidia}

Alienum est omne quicquid optando euenit.

28. Alienum nobis, nostrum plus aliis placet.

151. Id agas ne quis tuo te merito oderit.

165. Inuidiam ferre aut fortis aut foelix potest.

167. Inuidia tacite, sed minute irascitur.

\section{Prejuicios}

33. Ad poenitendum properat, cito qui iudicat.

128. Graue praeiudicium est, quod iudicium non habet.

172. In uindicando criminosa est celeritas.

\section{Molicie}

68. Bonarum rerum consuetudo pessima est.

127. Grauissimum est imperium consuetudinis.

207. Mala est uoluptas alienis assuescere.

\section{Desconfianza}

7. Ad tristem partem strenua suspicio.

27. Animo dolenti nil oportet credere.

107. Fides, ut anima, unde abiit, nunquam redit.

Desidia e indulgencia

35. Arcum intensio frangit, animum remissio.

86. Cui plus licet quam par est, plus uult quam licet.

\section{Ingratitud}

90. Dixeris maledicta cuncta, cum ingratum hominem dixeris.

161. Ingratus unus miseris omnibus nocet.

\section{Adulación}

106. Facilitas animi ad partem stultitiae rapit.

139. Habet suum uenenum blanda oratio.

\section{Hipocresía}

52. Bonitatis uerba imitari, maior malicia est. 


\section{Soberbia}

78. Cito ignominia, fit superbi gloria.

\section{Estupidez}

82. Contemni est leuius, quam stultitia percuti.

\section{Discordia}

95. Discordia fit charior concordia.

IV.- Estereotipos:

\section{La Mujer}

6. Aut amat, aut odit mulier, nihil est tertium.

20. Aperte cum est mala mulier, tum demum est bona.

77. Casta ad uirum matrona parendo imperat.

94. Dediscere flere foeminam, est mendacium.

113. Foeminae naturam regere, desperare est omnium.

206. Malo in consilio foeminae uincunt uiros.

217. Mulier cum sola cogitat, male cogitat.

222. Mulier quae nubit multis, multis non placet.

\section{La vejez}

24. Astute dum caelatur, se aetas indicat.

30. Anus cum ludit, morti delicias facit.

111. Frustra, cum ad senectam uentum est, repetes adolescentiam.

138. Heu quam poenitenda incurrunt uiuendo diu.

\section{Herencias y herederos}

146. Haeredem scire, utilius est quam quaerere.

147. Haeredis fletus, sub persona risus est.

205. Magis haeres fidus nascitur, quam scribitur.

214. Male secum agit aeger, medicum qui haeredem facit.

\section{Médicos y medicina}

73. Crudelem medicum intemperans aeger facit.

209. Mala est medicina, ubi aliquid naturae perit.

\section{El discípulo}

87. Discipulus est prioris, posterior dies.

149. Inferior horret, quicquid peccat superior.

\section{El borracho}

12. Absentem laedit, qui cum ebrio litigat.

\section{El dolor}

93. Dolor decrescit, ubi quo crescat non habet.

\section{El tahur}

34. Aleator quanto in arte est melior, tanto est nequior. 


\section{El charlatán}

83. Comes facundus, in uia pro uehiculo est.

\section{El hombre}

145. Homo uitae commodatus, non donatus est.

V.- No CLASIFICADOS:

9. Aspicere oportet, possis quod deperdere.

50. Coiunctio animi maximi est cognatio.

152. Inuitum cum retineas, exire incitas.

160. Impune peccat in eum, qui peccat rarior.

215. Minus decipitur, cui negatur celeriter.

226. Nil proprium ducas, quod mutari posset.

235. Quotidie est deterior, posterior dies. 